\title{
TLR8 regulation of $L I L R A 3$ in monocytes is abrogated in human immunodeficiency virus infection and correlates to CD4 counts and virus loads
}

Hui Zhi Low ${ }^{1 *}$ (D), Gerrit Ahrenstorf ${ }^{1}$, Claudia Pommerenke ${ }^{3}$, Nadine Habermann ${ }^{1}$, Klaus Schughart 3,4,5, David Ordóñez ${ }^{1}$, Renata Stripecke ${ }^{2}$, Esther Wilk ${ }^{3}$ and Torsten Witte ${ }^{1}$

\begin{abstract}
Background: LILRA3 is an immunostimulatory molecule which can conditionally induce the proliferation of cytotoxic cells. LILRA3 has a deletion genotype which is associated with multiple immune disorders. In this study, we wanted to analyze the regulation of LILRA3 and its significance in the context of HIV infection.

Results: We analyzed a panel of TLR agonists and found that SSRNA40, a TLR8 agonist, is a potent inducer of LILRA3 in healthy individuals. However, this regulation is much diminished in HIV. Comparison of TLR8 to TLR4 induction of LILRA3 indicated that LPS induces less LILRA3 than ssRNA40 among healthy controls, but not HIV patients. Levels of LILRA3 induction correlated to virus load and CD4 counts in untreated patients. Recombinant LILRA3 can induce a host of proinflammatory genes which include IL-6 and IL-1a, as well as alter the expression of MHC and costimulatory molecules in monocytes and B-cells.

Conclusion: Our experiments point towards a beneficial role for LILRA3 in virus infections, especially in ssRNA viruses, like HIV, that engage TLR8. However, the potentially beneficial role of LILRA3 is abrogated during a HIV infection. We believe that more work has to be done to study the role of LILRA3 in infectious diseases and that there is a potential for exploring the use of LILRA3 in the treatment of virus infections.
\end{abstract}

Keywords: LILRA3, TLR8, TLR4, Monocytes, HIV

\section{Background}

Extensive work has been done to functionally characterize members of the leukocyte immunoglobulin like receptor (LILR) family [1-11]. In comparison, the function of leukocyte immunoglobulin like receptor A3 (LILRA3; ILT6; CD85e) has been less well characterized. LILRA3 is a $70 \mathrm{kDa}$ highly glycosylated protein secreted by monocytes. The differentiation of monocytes into osteoclasts and dendritic cells has been shown to upregulate LILRA3 $[12,13]$. It is the only member of the LILR family that

\footnotetext{
*Correspondence: low.huizhi@mh-hannover.de

${ }^{1}$ Department of Clinical Immunology and Rheumatology, Hannover

Medical School, Carl-Neuberg-Str. 1, 30625 Hannover, Germany

Full list of author information is available at the end of the article
}

exists constitutively as a soluble protein due to a lack of a transmembrane domain and cytoplasmic tail. LILRA3 binds to HLA-G and classical HLAs with a much lower affinity than LILRB1 and LILRB2 [14], although it seems to bind to free heavy chain forms of HLA-C with higher affinity [15].

Around $30 \%$ of healthy Caucasians carry the LILRA3 deletion of $6,7 \mathrm{~Kb}$ which includes nearly the complete coding sequence $[8,16]$. The homozygous deletion is found in $3 \%$ of the healthy Caucasian population and confers susceptibility to some autoimmune diseases [1719], HIV-infection (in revision), and B cell non-hodgkin lymphoma [20].

Other members of the LILR family were demonstrated to play important roles in HIV infection [21]. Myeloid 
dendritic cells in elite controllers, who spontaneously maintain low viremia, have a selective upregulation of LILRB1 and LILRB3, whereas patients with a progressive infection showed a downregulation of LILRA2 and upregulation of LILRB2 [22].

As we have shown in a previous report, LILRA3 is an immunostimulatory molecule that specifically induces the proliferation of CD8 T-cells and NK-cells in the presence of allogeneic stimulation, as well as the production of pro-inflammatory cytokines [20]. Based on these properties, we suspect that LILRA3 could be involved in immune responses against viruses. However, nothing is known about the processes upstream of LILRA3 and their regulation.

Pattern recognition receptors (PRRs) are widely expressed on a variety of immune sensing cells. They recognize pathogen associated molecular patterns to initiate the innate immune response and direct adaptive immunity $[23,24]$. The best characterized PRRs are the toll-like receptors (TLR). Among the 10 members which sense a variety of infection types, those associated with virus infections are intracellular TLR3 (dsRNA), -7, -8 (both ssRNA) and -9 (unmethylated CpG DNA).

We used TLR-agonists as surrogates for whether virus infections could induce expression of LILRA3. We wanted to determine which TLR agonist could regulate LILRA3 expression and showed that ssRNA40, a TLR8 agonist, is a most prominent regulator of LILRA3. Since HIV is an ssRNA virus which has been documented to induce aberrant TLR responses in hosts [25, 26], we analyzed the TLR8 regulation of LILRA3 expression in the context of HIV infection.

\section{Results}

\section{TLR-8 induces LILRA3 expression in CD14 ${ }^{+}$monocytes}

In preliminary experiments, we subjected PBMCs from four donors to a panel of TLR agonist. Of the nine TLR agonists tested, a consistent immune response among the four donors, measured using IL6 expression, was only observed for Pam3Csk, heat-killed Listeria monocytogenes, LPS, flagellin, FSL-1 and ssRNA40. Three of the four donors had a substantial upregulation of LILRA3 to TLR8 agonist ssRNA40 (Fig. 1a), but we did not observe any obvious pattern of upregulation in LILRB1 and LILRA1 expression (Additional file 1: Figure S1). In order to confirm that TLR8 stimulation significantly upregulates LILRA3, we expanded the cohort using ssRNA40 stimulation, with ssRNA41 (similar to ssRNA40, but with uridine replaced with adenosine) as a control. Whereas ssRNA40 significantly upregulated the expression of LILRA3, ssRNA41 did not (Fig. 1b). LILRA3 could be detected in the supernatant of ssRNA40 stimulated LILRA3 positive PBMCs, but not in the negative controls and in the LILRA3 ${ }^{-/-}$donor, even though both donors were positively activated by ssRNA40 to secrete IL6 (Fig. 1c).

We determined monocytes to be the major producer of LILRA3 under ssRNA40 stimulation, as LILRA3 mRNA was detected only in $\mathrm{CD} 14^{+}$monocytes after 22-h ssRNA40 stimulation in PBMCs. In all three donors tested, almost all of the LILRA3 expression occurred in CD14+ monocytes, with two of the three donors expressing higher LILRA3 under ssRNA40 stimulation compared to LPS (Fig. 1d).

\section{LILRA3 is upregulated after other proinflammatory cytokines}

In order to determine the time point at which LILRA3 expression is the highest, we performed expression kinetics of LILRA3 using ssRNA40 stimulation of PBMCs. We also looked at the expression of other cytokines major cytokines as a comparison. Whereas the expression of the other cytokines tested peaked early at $4 \mathrm{~h}$ after ssRNA40 stimulation, LILRA3 expression only gradually increased to peak at $24 \mathrm{~h}$, after which its expression declined (Fig. 2a). In order to test of other cytokines were responsible for the expression, we neutralized IL-1R, TNF, IL-6R and IFN- $\gamma$ with their respective neutralization antibodies (Anakinra, Certolizumab, Tocilizumab and IFN- $\gamma$ neutralization antibody) to see if they had any effect on LILRA3 expression. No appreciable difference in ssRNA40-induced LILRA3 expression was observed even though expression of $I L-6$ was decreased in the presence of Anakinra and Certolizumab and increased in the presence of IFN- $\gamma$ neutralization (Fig. 2b). In summary, LILRA3 expression by TLR-8 stimulation peaks at $24 \mathrm{~h}$ and is independent from the expression of other immune relevant cytokines.

\section{TLR8 regulation of LILRA3 is abrogated in HIV infection}

We performed real time PCR for LILRA3 induction by 24-h incubation with ssRNA40 in genotyped HIV untreated patients, treated patients and healthy controls. Only samples of donors with at least one copy of the LILRA3 gene were analysed. Since monocytes are the major producers of LILRA3 and the percentages of monocytes could vary widely between persons, we performed the stimulation on isolated monocytes so that the results would not influenced by monocytic counts. Intra-group Mann-Whitney test between unstimulated control and ssRNA40 stimulation revealed that all three groups had a significant upregulation of LILRA3 expression upon ssRNA40 stimulation (Fig. 3a). However, Kruskal-Wallis with Dunn's multiple comparison test between the stimulated samples revealed that LILRA3 expression in untreated HIV patients was significantly 


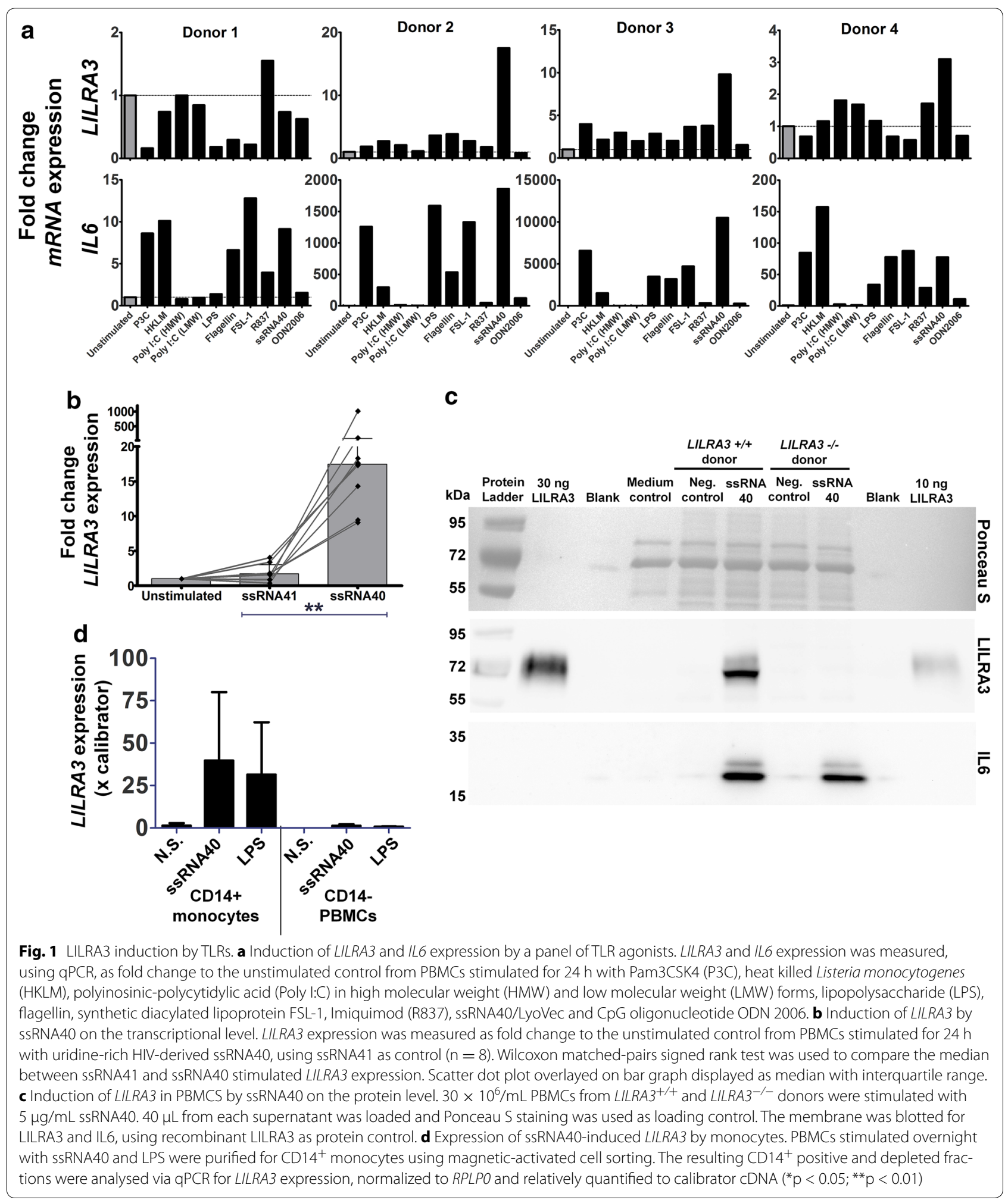

reduced compared to healthy controls, which is partially restored in treated patients (Fig. 3a). Interestingly, segregation of the patient and control groups into LILRA3 ${ }^{+/+}$ and $L I L R A 3^{+/-}$revealed higher LILRA3 expression among the LILRA3 ${ }^{+/+}$in healthy controls and HIV treated patients, although it was only significant among 


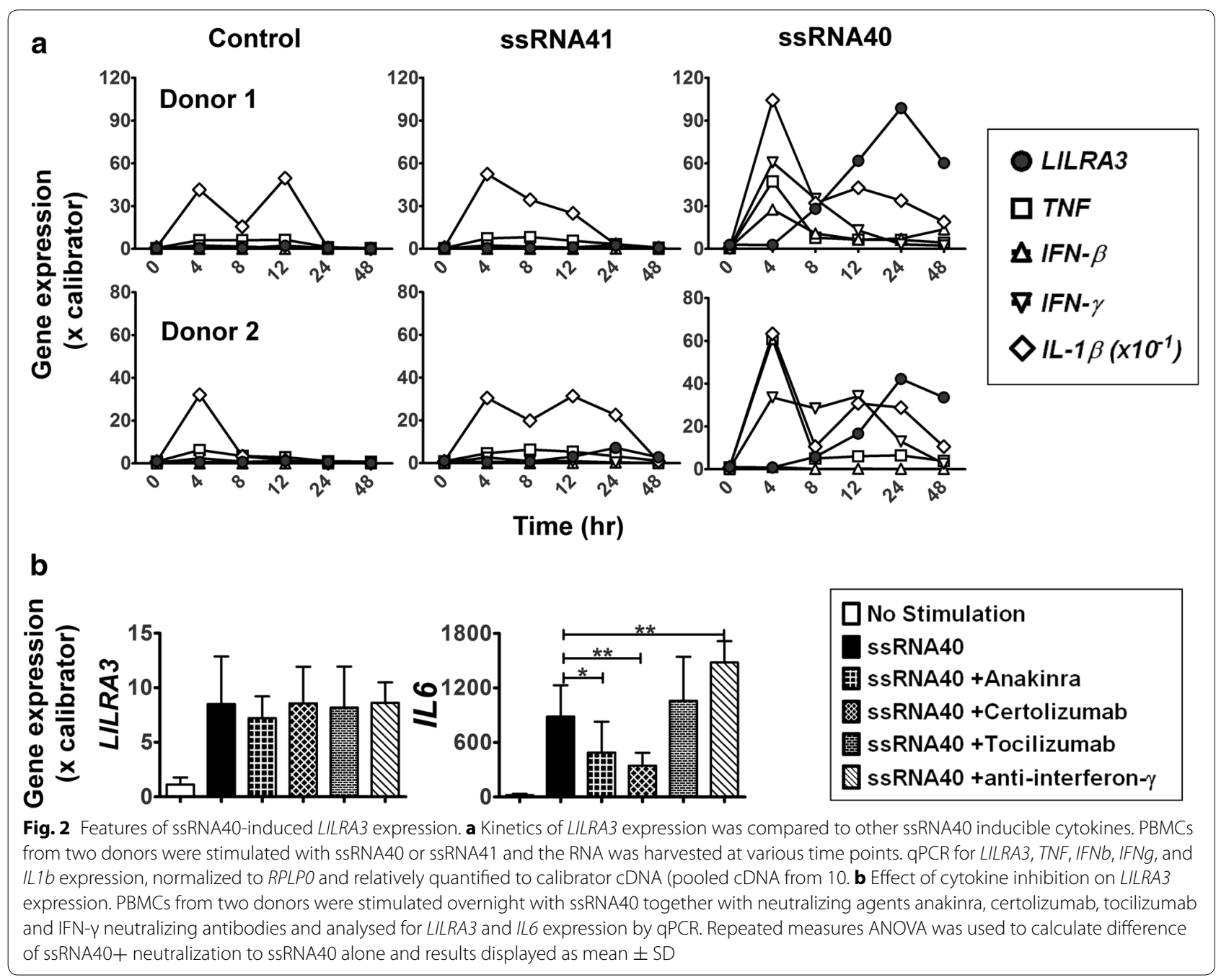

the healthy controls (Fig. 3b). Even among the LILRA3 $3^{+/+}$ samples, the HIV-untreated group had significantly lower LILRA3 expression than the healthy group and HIVtreated group.

HIV infection selectively abrogates ssRNA40, but not LPS, activation of LILRA3

As LPS seems to also induce LILRA3 (Fig. 1a), albeit more weakly, we compared if there were differences between the TLR8 and TLR4-mediated LILRA3 production. We performed the same experiment as before, with a new stimulus LPS for each sample. The second cohort confirmed the trend seen in the first cohort, with HIV untreated patients expressing less ssRNA40-induced LILRA3 compared to the healthy controls (Fig. 4a). However, after LPS stimulation, no significant difference was observed between healthy controls and HIV patients. Analysis of TNF expression via ELISA did not show a significant difference between healthy controls and
HIV patients after both ssRNA40 and LPS stimulation (Fig. 4a). Correlation of ssRNA40 versus LPS induction of LILRA3 expression gave striking differences. Among the healthy controls, there was a much higher expression of LILRA3 induced by ssRNA40 in comparison to LPS, with a slope of 1.69 using linear regression (Fig. 4b). However, this trend was reversed in HIV-untreated patients, with LILRA3 expression higher after LPS rather than ssRNA40 stimulation, with a much more gradual slope of 0.51 . Treated patients gave an intermediate trend, with a slope of 0.79 . Correlation analysis showed a statistically significant relationship between ssRNA40 and LPS induced LILRA3 expression for healthy donors and HIVuntreated donors.

\section{Both ssRNA40 and LPS-induced LILRA3 expression correlates with viral load and CD4 counts}

Since we believe that LILRA3 can be helpful in a virus infection due to its ability to induce proliferation in 

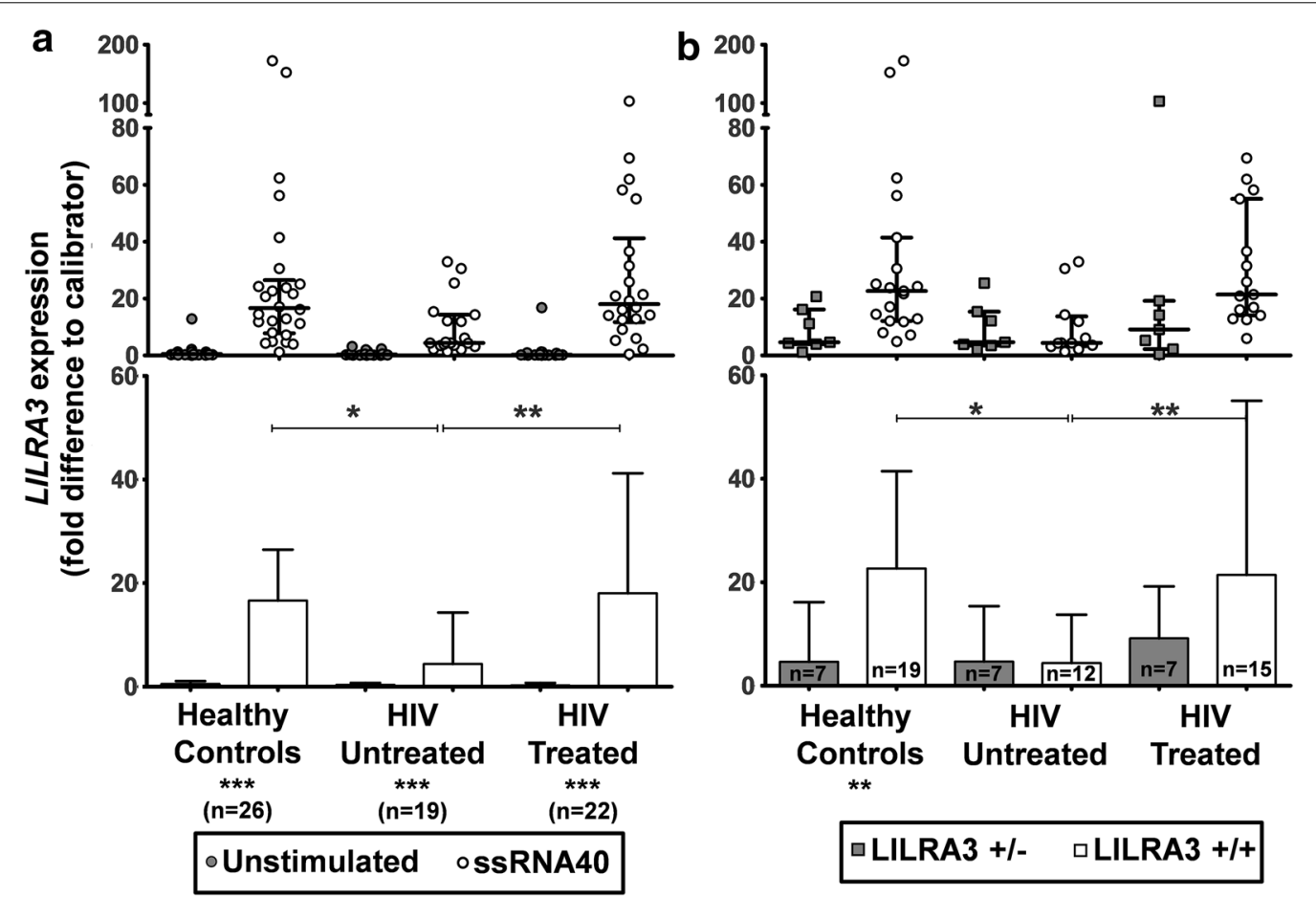

Fig. 3 Analysis of ssRNA40-induced LILRA3 expression in HIV. a Monocytes of healthy donors, HIV-untreated and HIV-treated patients were enriched using the Rosettesep system and stimulated overnight with ssRNA40 and analysed using qPCR for LILRA3. Wilcoxon matched-pairs signed rank test was used to compare intra-group unstimulated and ssRNA40-stimulated LILRA3 expression. Kruskal-Wallis test was used to compare inter-group ssRNA40-stimulated LILRA3 expression. Results displayed as median with interquartile range. b The values for the ssRNA40-induced LILRA3 (without unstimulated controls) were segregated according to genotype (LILRA3 ${ }^{+/+}$and $\left.L I L R A 3^{+/-}\right)$. Mann-Whitney test was used to compare intra-group LILRA3 expression. Kruskal-Wallis test was used to compare inter-group LILRA3 expression among LILRA3 ${ }^{+/+}$donors. Results displayed as median with interquartile range $\left({ }^{*} p<0.05 ;{ }^{* *} p<0.01 ;{ }^{* * *} p<0.001\right)$

cytotoxic cell subsets [20], we hypothesize that expression of LILRA3 could indicate better disease status of the patients, i.e. lower viral load, higher CD4 and CD8 T-cell and NK-cell counts. We performed a one-tailed Spearman correlation analysis of both ssRNA40 and LPSinduced LILRA3 expression with the abovementioned clinical parameters and observed that higher induction of LILRA3 was significantly correlated with lower viral load of HIV-untreated patients. Virus loads were not analysed for treated patients because they were almost uniformly below 20 virus copies/mL. LILRA3-induction correlated positively with CD4 T-cell count in untreated patients (Fig. 4c), but not for treated patients (Additional file 2: Figure S2).

Many immune activation genes are upregulated by LILRA3 To identify genes and pathways regulated by LILRA3, we analysed the gene expression signature induced by overnight incubation of PBMCs with recombinant LILRA3. Analysis of the microarray data revealed that among the top 50 genes upregulated by LILRA3, there appears to be a dominance of genes involved in immune activation, the foremost of which are the acute phase inflammatory cytokines IL- 6 and IL- $1 \alpha$ (Additional file 3: Figure S3A). The list of genes downregulated by LILRA3 however, paints a rather mixed picture, with a sprinkle of immunoregulatory genes like TREM2, CXCL11 and proteins involved in metabolic processes (e.g. CD36 and FABP4) or with less well known functions (e.g. PLBD1, LGALS2). Using gene set enrichment analysis (GSEA), we discovered a highly significant enrichment of several processes and pathways, among which chemotaxis, hematopoietic cell lineage pathways and genes involved in the pathogenesis of the autoimmune disorder rheumatoid arthritis (RA) had the highest number of annotated genes upregulated (Additional file 3: Figure S3B). Using realtime PCR, we confirmed the upregulation of IL6, ILIA and IL1B by LILRA3 (Fig. 5a). However, the consolidation of the results from the 4 different donors were confounded by the different optimum concentration of LILRA3 required 


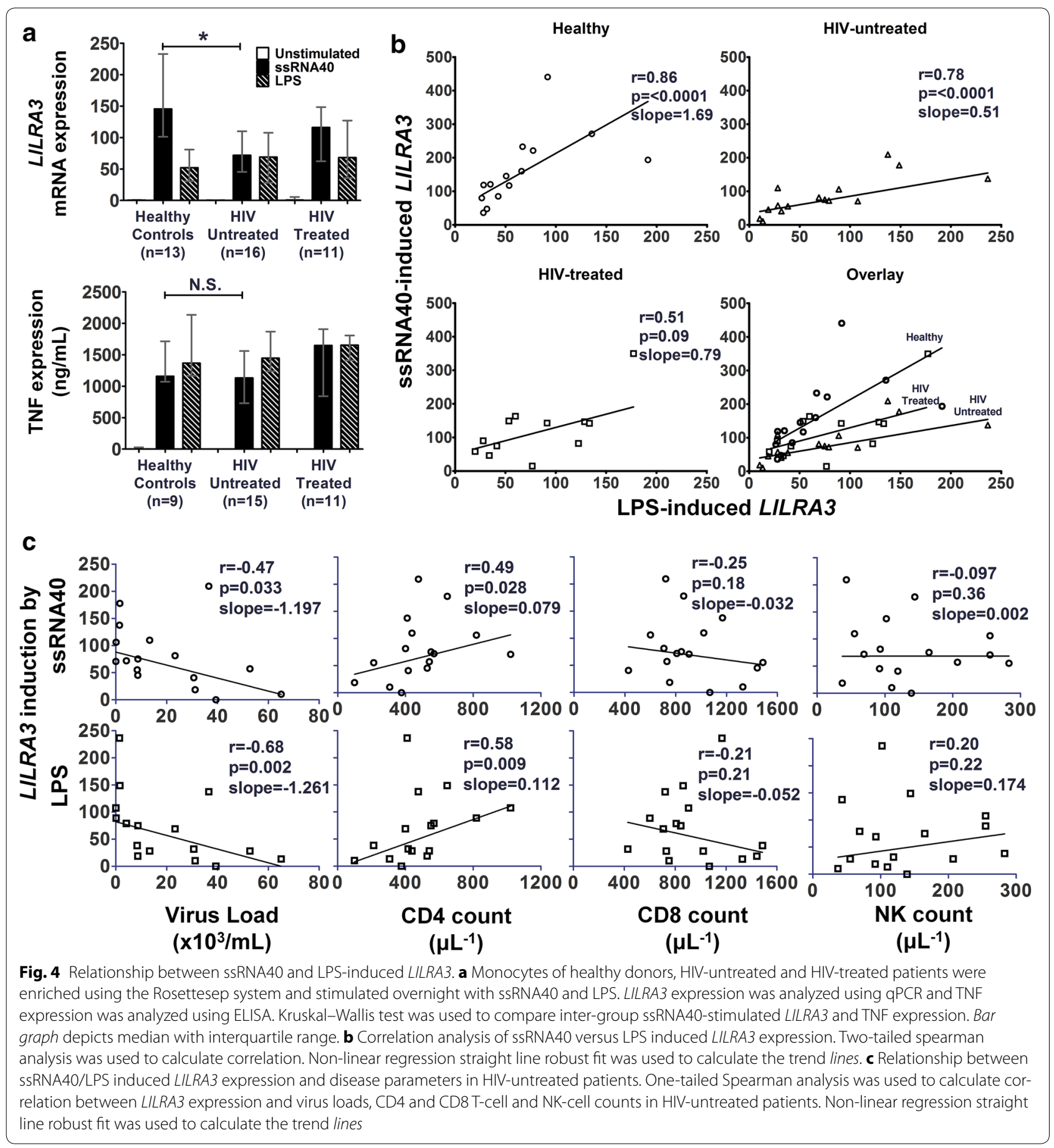

for stimulation for different donors, with donors 1 and 4 needing at least $1000 \mathrm{ng} / \mathrm{mL}$ LILRA3, while donors 2 and 3 requiring $500 \mathrm{ng} / \mathrm{mL}$ of LILRA3, beyond which the stimulation is abrogated. Purification from a mocktransfected expression did not lead to an increase of IL6 production like the purified LILRA3 (Additional file 4:
Figure S4). LILRA3 did not have much of an effect on LILRA3 itself and on IL10 (Fig. 5a).

\section{LILRA3 effects on the MHC and costimulatory molecules}

Since LILRA3 binds to an undetermined ligand expressed on the surface of monocytes and B-lymphocytes [20,27], 


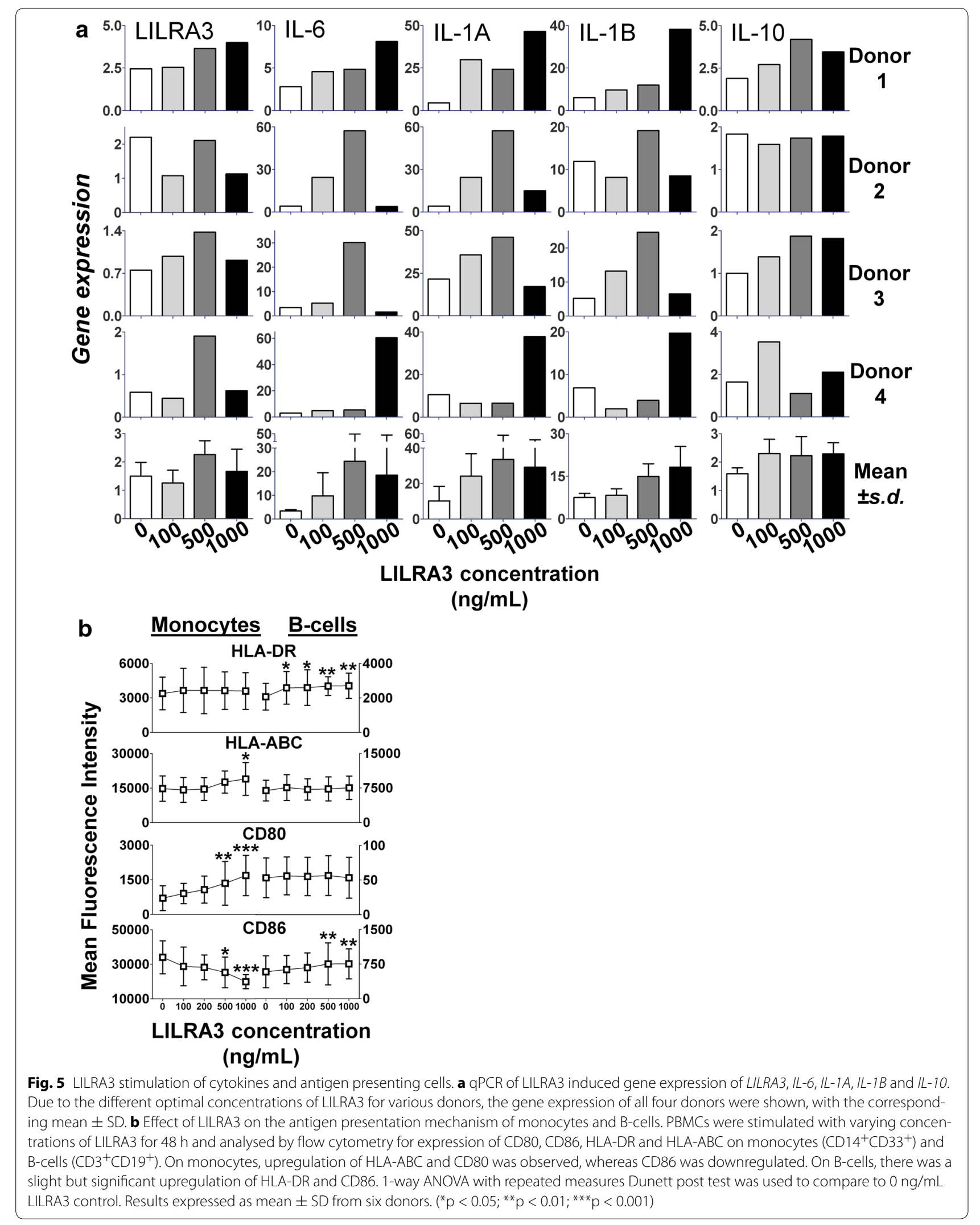


we tested if there is a direct effect of LILRA3 binding in the regulation of the MHC and costimulatory molecules expression levels on these cells. On monocytes, incubation with LILRA3 selectively upregulated HLA class I and CD80 surface expression. Notably, CD86 was conversely downregulated, whereas there was no significant change in HLA class II expression (Fig. 5b). On B-cells, there was a selective upregulation of HLA class II and CD86. However, no significant difference in the expression levels of HLA class I and CD80 were detected.

\section{Discussion}

TLRs and LILRs are two families of receptors which are widely and distinctly expressed on immune cells. Other members of the LILR family of receptors have been implicated in the control of TLR activity to several different bacterial infections (reviewed in [28]). The LILRs have also been shown play a role in viral infections like HIV [29-31]. We showed that ssRNA40, a TLR8-agonist, is the most prominent regulator of LILRA3 expression among the TLRs, and that this was mainly mediated by the monocyte population. ssRNA40 is a uridine-rich single stranded RNA sequence derived from the U5 region of HIV-1. Although ssRNA40 is an activating ligand for TLR7 in mice, in humans it has been shown to stimulate TLR8 responses in TLR8, but not TLR7, transfected HEK 293 cells [32]. Therefore, in our system, the ability of TLR7 and TLR9 to stimulate LILRA3 might be overlooked as the cell populations that express these receptors are underrepresented among the PBMCs. In human macrophages and dendritic cells, TLR3-ligand poly(I:C) has been shown to induce IFN-regulated genes, but not proinflammatory genes like TNF and IL6 [33], which might explain the missing IL6 response to poly(I:C) in our system.

Although LILRA3 expression peaks after $24 \mathrm{~h}$, neutralization of IL-1R, TNF, IL-6R and interferon- $\gamma$ did not lead to an appreciable change in LILRA3 (Fig. 2b), meaning that it is regulated independently of these cytokines. This could imply that LILRA3 expression is regulated directly by TLR 8 signalling pathways or by other indirect pathways/cytokines, for example type I interferons, which is as yet unknown. This should be of interest for further investigation.

In HIV-untreated patients, we observed a significant abrogation of the monocytic TLR8-induced LILRA3 in comparison to the robust response of healthy controls, as well as a reconstitution of this response in treated patients (Fig. 3). It is crucial to note that in this study, we do not measure the constitutive LILRA3 expression in HIV patients, but rather the strength of the LILRA3 response to TLR4 and TLR8 stimuli. This is more reflective of the immune status of the patients, rather than the constitutive expression due to viral loads. Nevertheless, the strength of the LILRA3 induction seems to be correlated with the viral load in untreated patients (Fig. 4c), suggesting a link between the strength of LILRA3 induction and the controlling of the infection. This would be consistent with previous studies showing that the strength of the TLR3, -7 and -8 responses are more robust among individuals who remain seronegative even after repeated sexual exposure to HIV [34], and that a mutant TLR8 allele which is more "functional" than the wild-type allele confers protection against HIV disease progression [35]. Considering that conventional dendritic cells also express TLR8 and are demonstrated play a role in control of HIV [36-38], it would be interesting to analyse their LILRA3 production in future experiments.

In order to assess whether the abrogation of LILRA3 expression in HIV infection is TLR8-specific or a general dampening of the monocytic response, we looked at the LPS induction of LILRA3 expression via TLR4 in a second cohort. ssRNA40-induced LILRA3 correlated well with LPS-induced LILRA3 expression across the three groups, suggesting that they act on similar signalling pathways to induce LILRA3 (Fig. 5b). However, while TLR8 stimulation gave a more robust LILRA3 response than TLR4 stimulation in healthy controls, this trend is paradoxically skewed towards LPS in HIV patients, who might actually benefit more from the TLR8-ssRNA response rather than the TLR4-LPS response. It is tempting initially to attribute this to immune dysregulation in chronically HIV-1 infected patients. However, the correlation between viral load and CD4 counts to LILRA3 expression that was present under TLR8 stimulation was even more strongly correlated under TLR4 stimulation (Fig. 4c), suggesting that the strength of the LPS response is also a factor in containing HIV-1. It has been shown that sensitization of monocytes with HIV-1 or TLR8-ligands led to higher production of proinflammatory cytokines by subsequent stimulation by LPS [39]. One explanation could be that because the TLR8 response was exhausted in these chronically HIV-1 infected patients, another TLR(s) was used as a compensatory mechanism. When we analyzed TNF production after ssRNA40 stimulation, we did not observe any significant decrease in TNF expression in HIV untreated patients, suggesting other factors at play other than TLR-8 exhaustion.

At this stage, it is still unclear whether the robustness of the LILRA3 induction is a cause or an effect of improved viral control and we cannot rule out in our study that the stronger LILRA3 responses are due to a general healthier immune status of the patients with lower viral loads. However, in light of a recent study of the genetics of LILRA3 deletion in HIV patients by Ahrenstorf et al. (in revision), HIV patients have higher percentages of 
$\operatorname{LILRA} 3^{-/-}$donors and there is a faster progression of the infection with decreasing gene dosage. Taken together, there is evidence to suggest that LILRA3 is helpful in immune control of HIV.

LILRA3 expression level is confirmed to be dependent on its gene-dosage, with $L I L R A 3^{+/-}$donors expressing substantially less LILRA3 than LILRA3 ${ }^{+/+}$donors (Fig. 4b). The untreated HIV patients had such depressed levels of LILRA3 induction that both genotypes gave similar low levels of gene expression. This is in line with a previous study that revealed decreased LILRA3 expression in LILRA3 $3^{+/-}$rheumatoid arthritis patients [40]. However, it is unclear whether this is the result of haploinsufficiency or the deletion allele being dominant negative.

Previously, we have shown that LILRA3 can induce a host of proinflammatory cytokines. We also show now in this paper that LILRA3 can upregulate transcription of $I L-6, I L-1 A$ and $I L-1 B$, but not $L I L R A 3$ itself and $I L-$ 10. Previous studies have shown that IL6 have suppressive effects on TNF production in vitro and in vivo [41, 42]. As LILRA3 upregulates IL6, but not TNF, the IL6 expression might have had repressive effects on TNF production, which could explain why Lee et al. [27] saw a repression of LPS-induced TNF by LILRA3. Interestingly, two out of the four donors we stimulated with LILRA3 responded best at $1000 \mathrm{ng} / \mathrm{mL}$, whereas the other 2 responded at $500 \mathrm{ng} / \mathrm{mL}$, but not at all at $1000 \mathrm{ng} / \mathrm{mL}$ (Fig. 5a). This means that LILRA3 activation is under very tight control, with different donors having different activation thresholds. The abrogation of its stimulatory effects at too high concentrations is most likely a mechanism against overactivation.

LILRA3 can modulate the expression $\mathrm{MHC}$ and costimulatory molecules in monocytes and B-cells. Although monocytes are the major producers of LILRA3 (Fig. 1d), the as yet unknown receptor for LILRA3 was found on monocytes and it exerts its immune modulating properties via the monocytes [20,27]. One possibility is that this is a mechanism for infected monocytes to alert nearby monocytes about the presence of virus particles in the vicinity.

Gene set enrichment analysis showed that LILRA3 regulates many genes involved in the pathology of RA (Additional file 3: Figure S3B). In the Han Chinese population, the genetic presence of LILRA3 is associated with increased risk of rheumatoid arthritis [40].

\section{Conclusion}

Our data point towards a role of LILRA3 in virus immunity. ssRNA40 is the most prominent inducer of LILRA3, which is produced by monocytes. Although HIV-1 patients express significantly less LILRA3 in response to ssRNA40, the LPS-induced LILRA3 remained unchanged. We also revealed that both TLR8 and TLR4 mediated LILRA3 expression can be correlated with viral loads and CD4 T-cell counts in untreated patients and that LILRA3 can upregulate proinflammatory cytokines, as well as induce changes MHC and costimulatory molecule expression monocytes and B-cells. Based on our findings, LILRA3 could potentially be exploited in the treatment of viral diseases like HIV.

\section{Methods \\ PBMC and monocyte isolation and culture}

PBMCs were isolated using density gradient centrifugation by layering blood onto Biocoll (Millipore) and centrifugation at $1200 \times g$ for $20 \mathrm{~min}$ without brake. The resulting buffy layer was then washed twice with PBS before use in subsequent experiments. Monocyte enrichment was performed using the RosetteSep ${ }^{\text {TM }}$ Human Monocyte Enrichment Cocktail (Stemcell Technologies). Positive enrichment of CD14 ${ }^{+}$monocytes was performed using CD14 Magnetic Particles (Becton-Dickinson). Cells were counted using trypan blue staining on a Neubauer hemocytometer and cultured at $37{ }^{\circ} \mathrm{C}$ with $5 \%$ $\mathrm{CO}_{2}$, in RPMI 1640 supplemented with $100 \mathrm{U} / \mathrm{mL}$ penicillin, $100 \mu \mathrm{g} / \mathrm{mL}$ streptomycin, $2 \mathrm{mM}$ L-glutamine (all Biochrom), and $10 \% \mathrm{AB}$ serum.

\section{RNA extraction and reverse transcription}

Total RNA was extracted using Zymo Research QuickRNA Microprep (Zymo Research), utilizing RNaseFree Dnase Set (Qiagen) for in-column genomic DNA removal. Reverse transcription was perform using High-Capacity cDNA Reverse Transcription Kit (Life Technologies).

\section{Quantitative real-time PCR}

Expression levels of LILRA3, TNF, IL- $1 \alpha$, IL-1 $\beta$, IFN- $\gamma$, IFN- $\beta$, IL- 6 were normalized to the housekeeping gene $60 \mathrm{~S}$ acidic ribosomal protein P0 (RPLP0) and relatively quantified using the $2^{-\mathrm{ddCt}}$ method either to the unstimulated control or to calibrator cDNA. Primer sequences are listed in Table 1. Calibrator cDNA was obtained from PBMCs ficoll purified from pooled blood of 10 healthy donors. Real-time PCR was performed using the Maxima SYBR Green/ROX qPCR Master Mix (Thermo Scientific) on the ABI PRISM 7000 Sequence Detection System (Applied Biosystems). The thermal cycling conditions composed of an initial denaturation step of $95^{\circ} \mathrm{C}$ for $10 \mathrm{~min}$, followed by 40 cycles of denaturation $\left(95^{\circ} \mathrm{C}\right.$ for $10 \mathrm{~s})$, annealing and $\left(60 / 57^{\circ} \mathrm{C}\right.$ for $\left.20 \mathrm{~s}\right)$ and extension/ detection $\left(72{ }^{\circ} \mathrm{C}\right.$ for $\left.40 \mathrm{~s}\right)$. 
Table 1 Real-time PCR primers

\begin{tabular}{|c|c|c|c|c|c|}
\hline \multirow{2}{*}{$\begin{array}{l}\text { Gene } \\
R P L P O\end{array}$} & \multicolumn{2}{|c|}{ Sequence $\left(5^{\prime}-3^{\prime}\right)$} & \multirow{2}{*}{$\begin{array}{l}\text { Annealing temp. }\left({ }^{\circ} \mathrm{C}\right) \\
60 / 57\end{array}$} & \multirow{2}{*}{$\begin{array}{l}\text { Length (bp) } \\
81\end{array}$} & \multirow{2}{*}{$\begin{array}{r}\text { Source } \\
(1)\end{array}$} \\
\hline & Fwd & TGCTTGATATCACAGAGGAAACTC & & & \\
\hline & Rev & CAATCTGCAGACAGACACTGG & & & \\
\hline \multirow[t]{2}{*}{ LILRA3 } & Fwd & AGGAGTGGGGACGTGACTT & 60 & 202 & (2) \\
\hline & Rev & GGTCTGGCACGGATCTGTC & & & \\
\hline \multirow[t]{2}{*}{ IFNB1 } & Fwd & ATTCTGCATTACCTGAAGGCCA & 60 & 141 & (1) \\
\hline & Rev & CCAGAGGCACAGGCTAGGA & & & \\
\hline \multirow[t]{2}{*}{ IFNG } & Fwd & TCGTTTTGGGTTCTCTTGGCT & 60 & 243 & (2) \\
\hline & Rev & TCTCCACACTCTTTTTGGATGCTC & & & \\
\hline \multirow[t]{2}{*}{ TNF } & Fwd & CTGGAAAGGACACCATGAGCA & 60 & 237 & (1) \\
\hline & Rev & GGGCCAGAGGGCTGATTAGA & & & \\
\hline \multirow[t]{2}{*}{ ILIA } & Fwd & AGAGGAAGAAATCATCAAGC & 57 & 122 & (3) \\
\hline & Rev & TTATACTTTGATTGAGGGCG & & & \\
\hline \multirow[t]{2}{*}{$I L 1 B$} & Fwd & TCTTTGAAGCTGATGGCCCTAAA & 60 & 188 & (1) \\
\hline & Rev & GAAGGTCTGTGGGCAGGGAA & & & \\
\hline \multirow[t]{2}{*}{ IL6 } & Fwd & GCAGAAAAAGGCAAAGAATC & 60 & 178 & (3) \\
\hline & Rev & CTACATTTGCCGAAGAGC & & & \\
\hline \multirow[t]{2}{*}{$\angle I L R B 1$} & Fwd & TTCTATGACAGAGTCTCCCTCTCGGT & 60 & 99 & (4) \\
\hline & Rev & AGTTTGCATCCATCCCTGTGACTG & & & \\
\hline \multirow[t]{2}{*}{ LILRA1 } & Fwd & ATCACAAAACAAGACTGCCTCACA & 60 & 81 & (1) \\
\hline & Rev & AGGACCAAGCCAGCTATGCC & & & \\
\hline
\end{tabular}

(1) NCBI Primer Blast; (2) Primer Bank; (3) Sigma Aldrich KiqStart; (4) Primer Quest

\section{Samples}

Permission for the study was obtained from the Institutional Review Board (IRB) of Hannover Medical School. Blood from HIV-1 chronically infected but untreated patients and HAART-treated patients with controlled viremia were obtained with written informed consent according to the Declaration of Helsinki from the HIV outpatient clinic of the Clinic for Immunology and Rheumatology, Hannover Medical School. Patient characteristics are listed under Table 2. Blood of healthy controls were obtained anonymously from blood donors at the Institute of Transfusion Medicine, Hannover

Table 2 Patient characteristics

\begin{tabular}{|c|c|c|c|c|}
\hline & $\begin{array}{l}\text { Healthy } \\
\text { controls }\end{array}$ & $\begin{array}{l}\text { HIV } \\
\text { untreated }\end{array}$ & $\begin{array}{l}\text { HIV } \\
\text { treated }\end{array}$ & $p$ value \\
\hline \multicolumn{5}{|l|}{ Cohort 1} \\
\hline Number & 26 & 19 & 22 & \\
\hline Mean age (SD) & $43.50(12.15)$ & $42.53(12.87)$ & $48.86(10.00)$ & $>0.05$ \\
\hline Male:female & $21: 5$ & $13: 6$ & $19: 3$ & $>0.05$ \\
\hline \multicolumn{5}{|l|}{ Cohort 2} \\
\hline Number & 13 & 16 & 11 & \\
\hline Mean age (SD) & $43.00(14.49)$ & $47.50(12.70)$ & $50.64(12.92)$ & $>0.05$ \\
\hline Male:female & $10: 3$ & $10: 6$ & $9: 2$ & $>0.05$ \\
\hline
\end{tabular}

Medical School as well as from volunteers from the laboratory.

\section{TLR-agonist stimulation experiments}

The Human TLR1-9 Agonist Kit (Invivogen) was used for the pilot study. $10^{6} / \mathrm{mL}$ PBMCs were stimulated for $24 \mathrm{~h}$ with $1 \mu \mathrm{g} / \mathrm{mL}$ Pam3CSK4 (P3C), $10^{8}$ cells $/ \mathrm{mL}$ heat killed Listeria monocytogenes (HKLM), $1 \mu \mathrm{g} / \mathrm{mL}$ polyinosinic-polycytidylic acid (Poly I:C) in high molecular weight (HMW) and low molecular weight (LMW) forms, $100 \mathrm{ng} / \mathrm{mL}$ lipopolysaccharide (LPS), $100 \mathrm{ng} / \mathrm{mL}$ flagellin, $100 \mathrm{ng} / \mathrm{mL}$ synthetic diacylated lipoprotein FSL-1, $1 \mu \mathrm{g} /$ $\mathrm{mL}$ iniquimod, $1 \mu \mathrm{g} / \mathrm{mL}$ ssRNA40/LyoVec and $5 \mu \mathrm{M}$ CpG oligonucleotide ODN 2006. For the kinetics, PBMC were stimulated with ssRNA40/Lyovec and ssRNA41 and harvested for real-time PCR analysis at various time points up to $48 \mathrm{~h}$. For the rest of the stimulation experiments, PBMCs or monocytes were stimulated overnight for 20-22 h with respective stimulants. In later samples, the supernatants from monocyte stimulation was analyzed for TNF production was analyzed using the TNF elisa kit (Biolegend). Anakinra (Sobi), Certolizumab (UCB) and Tocilizumab (Roche) were obtained as leftovers from clinical treatments and used to neutralize IL-1R, TNF and IL-6R respectively. Functional grade purified anti-human IFN gamma (eBioscience) was used to neutralize IFN- $\gamma$. 


\section{Western blot to detect LILRA3 secretion upon ssRNA40 stimulation}

Purified PBMCs from LILRA3 positive and deleted persons were cultured in Optimem $+0.5 \% \mathrm{AB}$-serum in the presence or absence of $5 \mu \mathrm{g} / \mathrm{mL}$ ssRNA40, using $3 \times 10^{6}$ cells per well per $100 \mu \mathrm{L}$ in a 96-well plate. After $48 \mathrm{~h}$ incubation, the supernatant was harvested, spun down at $13,000 \mathrm{rpm}$ for $5 \mathrm{~min}$ in a microcentrifuge and run on a 4-12\% reducing SDS-PAGE gel (Life Technologies), and transferred onto a nitrocellulose membrane. LILRA3 antibody clone 2E9 (Abnova) was used for detection, using Peroxidase Conjugated Stabilized Goat Anti-Mouse IgG $(\mathrm{H}+\mathrm{L})$ (Pierce) as secondary antibody. After stripping the membrane with Restore Western Blot Stripping Buffer (Thermo Scientific), IL6 was detected using clone MQ2-13A5 (Biolegend) together with Peroxidase Conjugated Stabilized Goat Anti-Rat IgG $(\mathrm{H}+\mathrm{L})$ (Pierce). SuperSignal West Dura Chemiluminescent Substrate (Pierce) was used in conjunction with Versadoc MP4000 (Biorad) to visualize the bands.

\section{DNA extraction and LILRA3 deletion genotyping}

Genomic DNA was extracted from whole blood using Quick-gDNA MiniPrep kit (Zymo Research) according to manufacturer's instructions. LILRA3 deletion genotyping was done by PCR using reverse primer 5'-GACAG CAGATTCTAAAACAGTG-3' for both genotypes; forward primers $5^{\prime}$-CCCCTGGAGCTCGTGG-3' for the presence genotype and $5^{\prime}$-CATCTCGATCTGCCACTGA CAC- $3^{\prime}$ for the deletion genotype. PCR for both genotypes was performed in multiplex using Amplitaq Gold DNA Polymerase (Applied Biosystems) using the following cycling conditions on the Biometra T3 thermocycler: initial denaturation at $95{ }^{\circ} \mathrm{C}$ for $20 \mathrm{~min}$, followed by 43 cycles of denaturation $\left(95{ }^{\circ} \mathrm{C}\right.$ for $\left.10 \mathrm{~s}\right)$, annealing and elongation $\left(62^{\circ} \mathrm{C}\right.$ for $2 \mathrm{~min}$ ) and a final elongation step of $62{ }^{\circ} \mathrm{C}$ for $10 \mathrm{~min}$. PCR products were analysed on a $1.5 \%$ agarose ethidium bromide gel for the presence (1150 bp) and deletion (250 bp) genotypes.

\section{Generation of recombinant LILRA3}

Mammalian LILRA3 was produced under a Lentivirus expression system. Briefly, LILRA3 cDNA was amplified by PCR using Phusion High Fidelity DNA Polymerase (Thermo Scientific) from total cDNA of PBMC from a LILRA $^{+/-}$donor using primers (Fwd: $5^{\prime}$-CGGACTAGT ATGACCCCCATCCTCACG-3'; Rev: 5' - CGACTAGTT CA ATGGTGATGGTGATGGTG CTCACCAGCCTTG GAGTC) incorporating SpeI digestion site at both ends and His-tag sequence at the $3^{\prime}$ end. The amplified LILRA3-His cDNA was cloned into the $\mathrm{XbaI}$ site in the multiple cloning site of pRRL-CMV-MCS-IRES-GFP [51], and the sequence verified by sequencing (MWG
Operon). The newly generated pRRL-CMV-LILRA3His-IRES-GFP was used to transfect HEK-293T cells using the LENTI-Smart INT kit (Invivogen) according to manufacturer protocol. Virus particles produced were concentrated using PEG-it Virus Precipitation Solution (System Biosciences) and used to transduce fresh 293T cells to generate stable LILRA3-His expressing cell line. A stably transduced cell line was also produced using of pRRL-CMV-MCS-IRES-GFP for the mock purification control. Mammalian LILRA3-His or its equivalent mock purification was produced by culturing the transduced cells using CELLine AD 1000 bioreactor flasks (INTEGRA Biosciences) and purifying the recombinant protein using HisPur Cobalt Resin from supernatant adjusted to pH 8.2-8.5. Endotoxin level was tested using LAL Chromogenic Endotoxin Quantitation Kit (Pierce) and determined to be virtually endotoxin free at $5 \times 10^{-6} \mathrm{EU} / \mu \mathrm{g}$ protein. LILRA3 purified from Baculovirus expression system was produced as described previously [20].

\section{LILRA3 stimulation experiments}

For microarray analysis, $10^{6} / \mathrm{mL}$ PBMCs were cultured for $24 \mathrm{~h}$ in the absence or presence of $1 \mu \mathrm{g} / \mathrm{mL}$ baculovirus LILRA3 in a $25 \mathrm{~cm}^{2}$ suspension culture flask. The cells were pelleted and their total RNA isolated using the Qiagen RNeasy Minikit according to the manufacturer's instructions. Microarray and data processing are detailed below and deposited in Gene Expression Omnibus (accession GSE61356). To confirm the microarray data, PBMCs were stimulated overnight with 100, 500 and $1000 \mathrm{ng} / \mathrm{mL}$ of mammalian LILRA3 and analyzed for $I L-6, I L-1 A, I L-1 B, I L-10$ and LILRA3 expression. For changes in B-cell and monocyte expression levels of $\mathrm{MHC}$ and costimulatory monecules, PBMCs incubated for $48 \mathrm{~h}$ with $0-1000 \mathrm{ng} / \mathrm{mL}$ baculovirus LILRA3 were stained with CD14-APC-CY7 (Biolegend), CD19-V450, CD3-V500, HLA-ABC-FITC or CD80-FITC, CD86-PE (all BD Bioscience) or HLA-DR-PE (Beckman Coulter), and analyzed via flow cytometry.

\section{Microarray experiment and data processing}

Cy3-labeled cRNA was synthesized using the one color Quick Amp Labeling kit (Agilent Technologies) according to the manufacturer's instructions. cRNA fragmentation and hybridization onto the Whole Human Genome Oligo Microarray V2 was carried out as recommended by the Agilent One-Color Microarray-Based Gene Expression Analysis Protocol V5.7. Data were analysed using the $\mathrm{R}$ software and several packages from BioConductor [43] and other sources. Preprocessing steps comprised background correction ("normexp"), quantile normalisation, probe summarisation, and $\log 2$ transformation. For the background correction, fitted intensities were 
calculated by the convolution of normal and exponential distributions to observed foreground and background intensities [44]. For a robust analysis, median values were calculated from replicate samples for each gene. Differentially expressed genes (DEG) were obtained by selecting genes with a minimal fold change of two and significant changes to untreated controls keeping false-discovery rate adjusted $\mathrm{p}$ values of $10 \%$ according to the rank products method in order to apply robust statistics for small replicate sizes $[45,46]$. In order to further functionally characterise the selected genes, GOstats for identifying gene ontology terms was applied [47]. A functional analysis of the selected gene list was done with SPIA, the signaling pathway impact analysis [48]. GSEA served for understanding the genome-wide expression of all genes [49] via cellHTS2 [50]. The data sets are available under GEO accession number GSE61356.

\section{Statistical Analysis}

All statistical analyses were performed using Graphpad Prism 5.

\section{Abbreviation LILRA3 leukocyte immunoglobulin like receptor A3}

\section{Additional files}

Additional file 1: Figure S1. Induction of LILRB1 and LILRA1 expression by a panel of TLR agonists.LILRA3 expression was measured, using qPCR, as fold change to the unstimulated control from PBMCs stimulated for 24 hours.

Additional file 2: Figure S2. Correlation between LILRA3 expression and CD4 and CD8 T-cell and NK-cell counts in HIV-treated patients. One-tailed Spearman analysis was used to calculate correlation. Virus loads were omitted because almost all treated patients had loads below detection limit.Spearman analysis was used to calculate correlation. Non-linear regression straight line robust fit was used to calculate the trend lines.

Additional file 3: Figure S3. Gene expression analysis of LILRA3 stimulated PBMCs. PBMCs from 5 healthy donors treated for 24 hours with or without $1 \mu \mathrm{g} / \mathrm{mL}$ LILRA3 was analysed for their gene expression using Whole Human Genome Oligo Microarray V2. (A) The top 50 up- and downregulated genes based on fold change in their expression levels between untreated controls and LILRA3 treatment. (B) and (C) GSEA map based on gene ontology for biological processes and KEGG pathway mapping respectively. Genes regulated by LILRA3 appears to be enriched for, among others, chemotaxis, rheumatoid arthritis and hematopoietic cell lineage pathways. Size of the nodes is representative of the number of annotated genes within the pathway/term that is regulated, whereas the connection thickness is representative of the number of overlapping genes between connected nodes.

Additional file 4: Figure S4. IL6 upregulation by purified mammalian his-tagged LILRA3, but not by a mock purification. PBMC stimulated for 24 hours by LILRA3 or an equivalent amount of a mock purification was analyzed for $1 L 6$ production by $\mathrm{qPCR}$.

\section{Authors' contributions}

TW, HZL participated in the conception of the project and experiments. HZL performed and analysed the bulk of the experiments. NH contributed to LILRA3 stimulation experiments on B-cells and monocytes. HZL and DOV contributed to drafting of the manuscript. RS provided the lentiviral vectors. $\mathrm{KS}, \mathrm{CP}$ and EW contributed to the analysis of microarray data. All authors read and approved the final manuscript.

\section{Author details}

${ }^{1}$ Department of Clinical Immunology and Rheumatology, Hannover Medical School, Carl-Neuberg-Str. 1, 30625 Hannover, Germany. ${ }^{2}$ Department of Hematology, Hemostasis, Oncology and Stem Cell Transplantation, Hannover Medical School, Hannover, Germany. ${ }^{3}$ Department of Infection Genetics, Helmholtz Centre for Infection Research, Braunschweig, Germany. ${ }^{4}$ University of Veterinary Medicine, Hannover, Germany. ${ }^{5}$ University of Tennessee Health Science Center, Memphis, TN, USA.

\section{Acknowledgements}

This work was supported by the Deutsche Forschungs Gemeinschaft grant to Klinische Forschergruppe 250, TP3, WI 1031/6-1 to Torsten Witte. Microarray raw data used or referred to in this publication were generated with much help from Oliver Dittrich-Breiholz and by the Research Core Unit Transcriptomics of Hannover Medical School.

\section{Competing interests}

The authors declare that they have no competing interests.

Received: 15 September 2015 Accepted: 29 February 2016

Published online: 12 March 2016

\section{References}

1. Cao W, Rosen DB, Ito T, Bover L, Bao M, Watanabe G, Yao Z, Zhang L, Lanier LL, Liu YJ. Plasmacytoid dendritic cell-specific receptor ILT7-Fc epsilonRI gamma inhibits Toll-like receptor-induced interferon production. J Exp Med. 2006;203:1399-405.

2. Cella M, Dohring C, Samaridis J, Dessing M, Brockhaus M, Lanzavecchia A, Colonna M. A novel inhibitory receptor (ILT3) expressed on monocytes, macrophages, and dendritic cells involved in antigen processing. J Exp Med. 1997;185:1743-51.

3. Chang CC, Ciubotariu R, Manavalan JS, Yuan J, Colovai Al, Piazza F, Lederman S, Colonna M, Cortesini R, Dalla-Favera R, Suciu-Foca N. Tolerization of dendritic cells by T(S) cells: the crucial role of inhibitory receptors ILT3 and ILT4. Nat Immunol. 2002;3:237-43.

4. Manavalan JS, Rossi PC, Vlad G, Piazza F, Yarilina A, Cortesini R, Mancini D, Suciu-Foca N. High expression of ILT3 and ILT4 is a general feature of tolerogenic dendritic cells. Transpl Immunol. 2003;11:245-58.

5. Navarro F, Llano M, Bellon T, Colonna M, Geraghty DE, Lopez-Botet M. The ILT2(LIR1) and CD94/NKG2A NK cell receptors respectively recognize HLA-G1 and HLA-E molecules co-expressed on target cells. Eur I Immunol. 1999;29:277-83.

6. Saverino D, Fabbi M, Ghiotto F, Merlo A, Bruno S, Zarcone D, Tenca C, Tiso M, Santoro G, Anastasi G, Cosman D, Grossi CE, Ciccone E. The CD85/ LIR-1/ILT2 inhibitory receptor is expressed by all human T lymphocytes and down-regulates their functions. J Immunol. 2000;165:3742-55.

7. Shiroishi M, Tsumoto K, Amano K, Shirakihara Y, Colonna M, Braud VM, Allan DS, Makadzange A, Rowland-Jones S, Willcox B, Jones EY, van der Merwe PA, Kumagai I, Maenaka K. Human inhibitory receptors Ig-like transcript 2 (ILT2) and ILT4 compete with CD8 for MHC class I binding and bind preferentially to HLA-G. Proc Natl Acad Sci USA. 2003;100:8856-61.

8. Torkar M, Haude A, Milne S, Beck S, Trowsdale J, Wilson MJ. Arrangement of the ILT gene cluster: a common null allele of the ILT6 gene results from a 6.7-kbp deletion. Eur J Immunol. 2000;30:3655-62.

9. Vlad G, Chang C-C, Colovai Al, Berloco P, Cortesini R, Suciu-Foca N. Immunoglobulin-like transcript 3: a crucial regulator of dendritic cell function. Hum Immunol. 2009;70:340-4. 
10. Willcox BE, Thomas LM, Chapman TL, Heikema AP, West AP, Bjorkman PJ. Crystal structure of LIR-2 (ILT4) at 1.8 A: differences from LIR-1 (ILT2) in regions implicated in the binding of the Human Cytomegalovirus class I MHC homolog UL18. BMC Struct Biol. 2002;2:6.

11. Li NL, Davidson CL, Humar A, Burshtyn DN. Modulation of the inhibitory receptor leukocyte Ig-like receptor 1 on human natural killer cells. Front Immunol. 2011;2:46

12. Arm JP, Nwankwo C, Austen KF. Molecular identification of a novel family of human Ig superfamily members that possess immunoreceptor tyrosine-based inhibition motifs and homology to the mouse gp49B1 inhibitory receptor. J Immunol. 1997;159:2342-9.

13. Jones DC, Roghanian A, Brown DP, Chang C, Allen RL, Trowsdale J, Young NT. Alternative mRNA splicing creates transcripts encoding soluble proteins from most LILR genes. Eur J Immunol. 2009;39:3195-206.

14. Ryu M, Chen Y, Qi J, Liu J, Fan Z, Nam G, Shi Y, Cheng H, Gao GF. LILRA3 binds both classical and non-classical HLA class I molecules but with reduced affinities compared to LILRB1/LILRB2: structural evidence. PLOS One. 2011;6:e19245

15. Jones DC, Kosmoliaptsis V, Apps R, Lapaque N, Smith I, Kono A, Chang C, Boyle LH, Taylor CJ, Trowsdale J, Allen RL. HLA class I allelic sequence and conformation regulate leukocyte lg-like receptor binding. J Immunol. 2011;186:2990-7.

16. Wilson MJ, Torkar M, Haude A, Milne S, Jones T, Sheer D, Beck S, Trowsdale J. Plasticity in the organization and sequences of human KIR/ILT gene families. Proc Natl Acad Sci USA. 2000;97:4778-83.

17. Kabalak G, Dobberstein SB, Matthias T, Reuter S, The YH, Dorner T, Schmidt RE, Witte T. Association of immunoglobulin-like transcript 6 deficiency with Sjogren's syndrome. Arthritis Rheum. 2009;60:2923-5.

18. Koch S, Goedde R, Nigmatova V, Epplen JT, Müller N, de Seze J, Vermersch P, Momot T, Schmidt RE, Witte T. Association of multiple sclerosis with ILT6 deficiency. Genes Immun. 2005;6:445-7.

19. Ordonez D, Sanchez AJ, Martinez-Rodriguez JE, Cisneros E, Ramil E, Romo N, Moraru M, Munteis E, Lopez-Botet M, Roquer J, Garcia-Merino A, Vilches C. Multiple sclerosis associates with LILRA3 deletion in Spanish patients. Genes Immun. 2009;10:579-85.

20. Low HZ, Reuter S, Topperwien M, Dankenbrink N, Peest D, Kabalak G, Stripecke R, Schmidt RE, Matthias T, Witte T. Association of the LILRA3 deletion with B-NHL and functional characterization of the immunostimulatory molecule. PLoS One. 2013;8:e81360.

21. Lichterfeld M, Yu XG. The emerging role of leukocyte immunoglobulinlike receptors (LILRs) in HIV-1 infection. J Leukoc Biol. 2012;91:27-33.

22. Huang J, Burke PS, Cung TD, Pereyra F, Toth I, Walker BD, Borges L, Lichterfeld M, Yu XG. Leukocyte immunoglobulin-like receptors maintain unique antigen-presenting properties of circulating myeloid dendritic cells in HIV-1-infected elite controllers. J Virol. 2010;84:9463-71.

23. Iwasaki A, Medzhitov R. Toll-like receptor control of the adaptive immune responses. Nat Immunol. 2004;5:987-95.

24. Mogensen TH. Pathogen recognition and inflammatory signaling in innate immune defenses. Clin Microbiol Rev. 2009;22:240-73.

25. Heggelund L, Müller F, Lien E, Yndestad A, Ueland T, Kristiansen KI, Espevik T, Aukrust P, Frøland SS. Increased expression of toll-like receptor 2 on monocytes in HIV infection: possible roles in inflammation and viral replication. Clin Infect Dis. 2004;39:264-9.

26. Lester RT, Yao X-D, Ball TB, McKinnon LR, Omange WR, Kaul R, Wachihi C, Jaoko W, Rosenthal KL, Plummer FA. HIV-1 RNA dysregulates the natural TLR response to subclinical endotoxemia in kenyan female sex-workers. PLOS One. 2009:4:e5644.

27. Lee THY, Mitchell A, Lau SL, An H, Rajeaskariah P, Wasinger V, Raftery M, Bryant K, Tedla N. Glycosylation in a mammalian expression system is critical for the production of functionally active leukocyte immunoglobulinlike receptor A3 protein. J Biol Chem. 2013. doi:10.1074/jbc.M113.478578.

28. Pilsbury LE, Allen RL, Vordermeier M. Modulation of toll-like receptor activity by leukocyte Ig-like receptors and their effects during bacterial infection. Mediators Inflamm. 2010;2010:e536478.

29. Bashirova AA, Martin-Gayo E, Jones DC, Qi Y, Apps R, Gao X, Burke PS, Taylor CJ, Rogich J, Wolinsky S, Bream JH, Duggal P, Hussain S, Martinson J, Weintrob A, Kirk GD, Fellay J, Buchbinder SP, Goedert JJ, Deeks SG, Pereyra F, Trowsdale J, Lichterfeld M, Telenti A, Walker BD, Allen RL, Carrington M, YU XG. LILRB2 interaction with HLA class I correlates with control of HIV-1 infection. PLoS Genet. 2014;10:e1004196.
30. O'Connor GM, Holmes A, Mulcahy F, Gardiner CM. Natural Killer cells from long-term non-progressor HIV patients are characterized by altered phenotype and function. Clin Immunol. 2007;124:277-83.

31. Anfossi N, Doisne J-M, Peyrat M-A, Ugolini S, Bonnaud O, Bossy D, Pitard V, Merville P, Moreau J-F, Delfraissy J-F, Dechanet-Merville J, Bonneville M, Venet A, Vivier E. Coordinated expression of Ig-like inhibitory MHC class I receptors and acquisition of cytotoxic function in human CD8+ T cells. J Immunol. 1950;2004(173):7223-9.

32. Heil F, Hemmi H, Hochrein H, Ampenberger F, Kirschning C, Akira S, Lipford G, Wagner H, Bauer S. Species-specific recognition of single-stranded RNA via toll-like receptor 7 and 8. Science. 2004;303:1526-9.

33. Lundberg AM, Drexler SK, Monaco C, Williams LM, Sacre SM, Feldmann M, Foxwell BM. Key differences in TLR3/poly I:C signaling and cytokine induction by human primary cells: a phenomenon absent from murine cell systems. Blood. 2007;110:3245-52.

34. Biasin M, Piacentini L, LoCaputo S, Naddeo V, Pierotti P, Borelli M, Trabattoni D, Mazzotta F, Shearer GM, Clerici M. TLR activation pathways in HIV-1-exposed seronegative individuals. J Immunol. 2010;184:2710-7.

35. Oh D-Y, Taube S, Hamouda O, Kücherer C, Poggensee G, Jessen H, Eckert JK, Neumann K, Storek A, Pouliot M, Borgeat P, Oh N, Schreier E, Pruss A, Hattermann K, Schumann RR. A functional toll-like receptor 8 variant is associated with HIV disease restriction. J Infect Dis. 2008;198:701-9.

36. Martinson JA, Roman-Gonzalez A, Tenorio AR, Montoya CJ, Gichinga CN, Rugeles MT, Tomai M, Krieg AM, Ghanekar S, Baum LL, Landay AL. Dendritic cells from HIV-1 infected individuals are less responsive to toll-like receptor (TLR) ligands. Cell Immunol. 2007;250:75-84.

37. Martin-Gayo E, Buzon MJ, Ouyang Z, Hickman T, Cronin J, Pimenova D, Walker BD, Lichterfeld M, Yu XG. Potent cell-intrinsic immune responses in dendritic cells facilitate HIV-1-specific T cell immunity in HIV-1 elite controllers. PLoS Pathog. 2015;11:e1004930.

38. Macatonia SE, Lau R, Patterson S, Pinching AJ, Knight SC. Dendritic cell infection, depletion and dysfunction in HIV-infected individuals. Immunology. 1990;71:38-45.

39. Mureith MW, Chang JJ, Lifson JD, Ndung'U T, Altfeld M. Exposure to HIV-1 encoded TLR8 ligands enhances monocytes response to microbial encoded TLR2/4 ligands. AIDS Lond Engl. 2010;24:1841-8.

40. Du Y, Cui Y, Liu X, Hu F, Yang Y, Wu X, Liu X, Ma X, Zuo X, Sheng Y, Liu X, Xu J, Zhu P, Sun L, Hong N, Zhang $X$, Guo J, Li Z. Contribution of functional LILRA3, but not nonfunctional LILRA3, to sex bias in susceptibility and severity of anti-citrullinated protein antibody-positive rheumatoid arthritis. Arthritis Rheumatol. 2014;66:822-30.

41. Aderka D, Le JM, Vilcek J. IL-6 inhibits lipopolysaccharide-induced tumor necrosis factor production in cultured human monocytes, U937 cells, and in mice. J Immunol. 1950;1989(143):3517-23.

42. Schindler R, Mancilla J, Endres S, Ghorbani R, Clark SC, Dinarello CA. Correlations and interactions in the production of interleukin-6 (IL-6), IL-1, and tumor necrosis factor (TNF) in human blood mononuclear cells: IL-6 suppresses IL-1 and TNF. Blood. 1990;75:40-7.

43. Gentleman RC, Carey VJ, Bates DM, Bolstad B, Dettling M, Dudoit S, Ellis B, Gautier L, Ge Y, Gentry J, Hornik K, Hothorn T, Huber W, lacus S, Irizarry R, Leisch F, Li C, Maechler M, Rossini AJ, Sawitzki G, Smith C, Smyth G, Tierney L, Yang JY, Zhang J. Bioconductor: open software development for computational biology and bioinformatics. Genome Biol. 2004;5:R80.

44. Ritchie ME, Silver J, Oshlack A, Holmes M, Diyagama D, Holloway A, Smyth GK. A comparison of background correction methods for two-colour microarrays. Bioinformatics. 2007;23:2700-7.

45. Breitling R, Armengaud P, Amtmann A, Herzyk P. Rank products: a simple, yet powerful, new method to detect differentially regulated genes in replicated microarray experiments. FEBS Lett. 2004;573:83-92.

46. van Iterson M, Hoen PA, Pedotti P, Hooiveld GJ, den Dunnen JT, van Ommen GJ, Boer JM, Menezes RX. Relative power and sample size analysis on gene expression profiling data. BMC Genomics. 2009;10:439.

47. Falcon S, Gentleman R. Using GOstats to test gene lists for GO term association. Bioinformatics. 2007;23:257-8.

48. Tarca AL, Draghici S, Khatri P, Hassan SS, Mittal P, Kim JS, Kim CJ, Kusanovic JP, Romero R. A novel signaling pathway impact analysis. Bioinformatics. 2009;25:75-82.

49. Subramanian A, Tamayo P, Mootha VK, Mukherjee S, Ebert BL, Gillette MA, Paulovich A, Pomeroy SL, Golub TR, Lander ES, Mesirov JP. Gene set enrichment analysis: a knowledge-based approach for 
interpreting genome-wide expression profiles. Proc Natl Acad Sci USA. 2005;102:15545-50

50. Boutros M, Bras LP, Huber W. Analysis of cell-based RNAi screens. Genome Biol. 2006;7:R66
51. Stripecke R, Koya RC, Ta HQ, Kasahara N, Levine AM. The use of lentiviral vectors in gene therapy of leukemia: combinatorial gene delivery of immunomodulators into leukemia cells by state-of-the-art vectors. Blood Cells Mol Dis. 2003;31:28-37.
Submit your next manuscript to BioMed Central and we will help you at every step:

- We accept pre-submission inquiries

- Our selector tool helps you to find the most relevant journal

- We provide round the clock customer support

- Convenient online submission

- Thorough peer review

- Inclusion in PubMed and all major indexing services

- Maximum visibility for your research

Submit your manuscript at www.biomedcentral.com/submit
(OioMed Central 